\title{
Automatic Generation of Quizzes from DBpedia According to Educational Standards
}

\author{
Oscar Rodríguez Rocha \\ University Côte d'Azur, CNRS, Inria, I3S, France \\ oscar.rodriguez-rocha@inria.fr
}

\begin{abstract}
Educational quizzes are a powerful and popular tool to test the knowledge acquired by a learner and also to deepen her/his knowledge about a specific subject in an informal and entertaining way. Their production is a time-consuming task that can be automated by taking advantage of existing knowledge bases available on the Web of Linked Open Data (LOD). For these quizzes to be useful to learners, they must be generated according to the knowledge and skills defined by official educational standards for each subject and school year. This paper shows an approach to generate quizzes automatically according to the official French educational standards, from two different knowledge bases. Likewise, we show an evaluation of both knowledge bases.
\end{abstract}

\section{CCS CONCEPTS}

- Computing methodologies $\rightarrow$ Knowledge representation and reasoning; • Applied computing $\rightarrow$ E-learning; • Information systems $\rightarrow$ Web data description languages;

\section{KEYWORDS}

eEducation, Semantic Web, Linked Data

\section{ACM Reference Format:}

Oscar Rodríguez Rocha and Catherine Faron Zucker. 2018. Automatic Generation of Quizzes from DBpedia According to Educational Standards. In The 2018 Web Conference Companion, April 23-27, 2018, Lyon, France. ACM, New York, NY, USA, 7 pages. https://doi.org/10.1145/3184558.3191534

\section{INTRODUCTION}

Educational quizzes have become a popular tool that allows students or learners to discover and deepen their knowledge about a specific subject in an informal and entertaining way. According to the authors in [15], their generation can be automated taking advantage of Semantic Web technologies and the Linked Open Data (LOD). However, being able to generate quizzes from resources of the LOD about a specific subject with questions relevant to the educational level of each student, is still an open research problem.

In this context, the research work described in this paper provides an answer to the following research questions:

(1) How to select resources from the LOD on a specific subject and relevant to a specific school level?

This paper is published under the Creative Commons Attribution 4.0 International (CC BY 4.0) license. Authors reserve their rights to disseminate the work on their personal and corporate Web sites with the appropriate attribution.

WWW'18 Companion, April 23-27, 2018, Lyon, France

(C) 2018 IW3C2 (International World Wide Web Conference Committee), published under Creative Commons CC BY 4.0 License.

ACM ISBN 978-1-4503-5640-4/18/04.

https://doi.org/10.1145/3184558.3191534

\author{
Catherine Faron Zucker \\ University Côte d'Azur, CNRS, Inria, I3S, France \\ faron@unice.fr
}

(2) Which referential of knowledge and skills is the most appropriate for the selection of suitable resources from the LOD?

(3) Which approach to use for the generation of quizzes about a subject, that takes into account the school level of a student?

In this research work, the use of a referential of knowledge and skills for the selection of relevant resources according to the existing school levels in France is considered. For this, we evaluate two existing educational references to determine which one allows to select the most relevant LOD resources from DBpedia France of a given subject and for different school courses. Finally, we describe an approach on how to perform the generation of the quizzes based on the selected referential.

The remainder of this paper is structured as follows: In section 2, we present the related works. In section 3, we present two structured knowledge bases that can be used as a standard of the required knowledge and skills in the French educational system by school year. In section 4, we describe how, by analyzing such knowledge bases, we can generate a knowledge graph from DBpedia, with resources that can be useful to produce quizzes automatically. In section 5 , we briefly describe the principle of the automatic generation of quizzes from such knowledge graphs. In section 6, we describe the implementation and evaluation of the knowledge graphs and the quizzes generated from them. Conclusions and future work are presented in Section 7.

\section{RELATED WORK}

Related works deal with the development of ontologies for the e-Education and curriculum and the generation of quizzes.

\subsection{Related works about ontologies for the e-Education and curriculum}

In [17], the authors provide a literature survey of the development and use of ontologies in the domain of e-learning systems. Among such works, CURONTO [5] is "an ontological model designed for the management of a curriculum and to facilite program review and assessment". However, it does not provide a description or representation of the knowledge and skills required by an educational system.

Gescur [11] is a tool dedicated to the management and evaluation of the implementation of the curriculum, which facilitates the curriculum management process". It relies on an ontology of concepts relevant to curriculum management in Secondary Schools, such as teachers, departments, objectives, subjects, modules, tasks, documents, policies, activities, learning objects, quality criteria, etc. This tool does not focus on the management of the knowledge and skills in educational systems. 
An educational semantic web ontology is proposed in [9]. It focuses on representing higher education concepts and assisting specialized e-learning systems.

In [13], the authors propose OntoEdu, an educational platform architecture for e-Learning which relies on an activity ontology that describes all the education activities and the relations among them, and a material ontology which describes the educational content organization. Unfortunately this ontology is not available online.

Finally, in [16], the authors present EduProgression, an ontological model for educational progressions, formalized in OWL, which is based on the modeling of the knowledge and skills of an educational system according to its different educational levels. Likewise this ontology is currently populated with the progressions of the French educational system for the subjects of History, Geography and Experimental Sciences and Technology.

\subsection{Related works about quizzes generation}

A set of strategies for the generation of questions, answers and distractors from a given ontology is proposed in [14]. The strategies proposed in this work are classified into three categories: classbased, property-based and terminology-based. As it will be explained, our automatic generation of quizzes was inspired on this work. Furthermore, we proposed a new classification of the strategies to generate quizzes that authors propose.

In [10], the authors present an approach for automatically generating computer-assisted assessments (CAA) from Semantic Webbased domain ontologies. They propose the addition of annotations to the meta-ontology used for the generation of questions and the addition of a semantic interpretation of the mapping between the domain ontology and the target question ontology. The semantic interpretation is based on the notion of question templates found on the BloomâĂŹs taxonomy of educational objectives.However, this work does not take into account the generation of questions oriented to a specific educational level.

The OntoQue system is dedicated to the generation of objective assessment items based on domain ontologies [4]. It uses knowledge inherent in the ontology about classes, properties, and individuals to generate semantically correct assessment items. Its authors evaluated the system with four OWL ontologies from different domains. Unlike this work, we propose a broader automatic approach that exploits the knowledge present in DBpedia, and which can be adapted to other knowledge bases or ontologies. In addition, our approach is based on the use of an educational reference to generate more specific questions according to the level of the learner.

In [12], the authors propose an approach to generate educational assessment items using Linked Open Data (DBpedia). They describe a streamline to create variables and populate simple choice item models using the IMS-QTI standard. A set of specific categories common to a set of resources is statically selected, from which questions, answers and distractors will be generated using SPARQL queries. Unlike our work prooposed in this paper, this work is not based on any educational reference and also does not propose an automatic approach.

In [2], the authors present an approach for the validation of ontologies which abstracts the complexity of formal languages by using a set of questions and answers to which the expert is subjected. The aim is to validate the conceptualization of the domain. Automatic reasoning and verbalization techniques are used to transform the facts present in an ontology into questions expressed in natural language, to be evaluated by a domain expert. The answer to each of these questions is then processed to automatically validate or correct the ontology. Unlike this approach, ours has not (yet) been evaluated for the validation of domain ontologies.

In [3], the authors present an approach to the automatic generation of Multiple Choice Questions (MCQs), based on the unsupervised Relation Extraction technique used in Natural Language Processing. It aims to identify the most important named entities and terminology in a document and then recognize semantic relations between them, without any prior knowledge as to the semantic types of the relations or their specific linguistic realization. In contrast, our approach is not based only domain ontologies, but it also allows to describe and define a specific domain of knowledge and exploit the knowledge found in knowledge bases such as DBpedia for the automatic generation of questions following educational references.

In [6-8], the authors present an approach to automatically generate multiple-choice questions from OWL ontologies. They describe a psychologically-plausible theory based on similarity [10] to control the difficulty of questions. They also describe a protocol to evaluate the characteristics of the generated questions such as the question difficulty. Unlike this work, we have not yet considered the determination of the difficulty of the generated questions. Without a doubt, this is an aspect that we will deal with in future works.

\section{EDUCATIONAL REFERENCES OF KNOWLEDGE AND SKILLS}

\subsection{EduProgression}

EduProgression [16], is an ontological model formalized in the standard Ontology Web Language (OWL) for the formalization of educational progressions or programs as defined in the French common bases of 2006 [1] and 2016. In this formalization, we identified the following main classes:

- Element of Knowledge and Skills (EKS). As skills and knowledge are keystones of the common bases, this element is the key concept of our model. It is represented by the EKS class, which is the central class of the EduProgression ontology. This class is common for the two releases of the common base.

An element of knowledge and skills is associated to a set of knowledge pieces (class Knowledge) and/or skills (class Skill) for a specific French school cycle (class Cycle) or course (class Course) that may contain reference points (class Point0fReference) and also a vocabulary of associated terms (class Vocabulary). More precisely:

- Knowledge. Instances of class Knowledge are also instances of skos:Concept and each one belongs to a skos: ConceptScheme that contains all the knowledge pieces of a given progression. An instance of EKS is related to an instance of Knowledge through the property hasknowledge. 
- Skill. An instance of EKS is related to an instance of Skill through property hasSkill.

- Course. In the French common base of 2006, the skills that students are expected to develop are defined by cycle and each cycle is organized into courses. For example, "the consolidation cycle" includes "the second year elementary course" (CE2), "the first-year intermediate course" (CM1) and "the second year intermediate course" (CM2). In this context, an instance of class Course represents a course in a cycle. An instance of EKS is related to an instance of Course through property hasCourse.

- Cycle. In the French common base of 2016, the skills that students are expected to develop are defined only by cycle. An instance of EKS is related to an instance of Cycle through property hascycle.

- PointOfReference. An instance of class PointOfReference represents an educational reference element on a specific element of knowledge and skills (an instance of EKS). An instance of EKS is related to an instance of class PointOfReference through property hasPointOfReference.

- VocabularyItem. Each element of knowledge and skills has vocabulary items. This vocabulary is compatible for the two common bases. An instance of EKS is related to an instance of class VocabularyItem through property hasVocabularyItem. A vocabulary item is also an instance of skos: Concept and it is related to an instance of skos: ConceptScheme which gathers the concepts of the thesaurus of the progression.

- Progression. In the common base released in 2006, the progressive acquisition of knowledge and skills is defined as a "progression", while for the current common base, the progressive acquisition of skills is defined as a "program". Therefore, a progression or a program in our model, is represented as an instance of the class Progression. It can be associated to an existing learning domain (through property hasLearningDomain) and to one or several EKSs (through property hasEKS).

- Learning domain. A learning domain represents a school subject like History or Mathematics. The learning domain is represented, in the ontology EduProgression, by an instance of the LearningDomain class, and it is also an instance of skos: Concept that is part of (only) one skos: ConceptScheme containing the only learning domains of a progression. Also, as they are SKOS concepts, learning domains are hierarchically organized by using the skos: broader and/or skos: narrower properties. A learning domain can be associated to a Progression or an EKS.

- Skills Domain. For the common base of 2016, each domain of skills can be represented in our model by an instance of the class SkillsDomain. An EKS can be associated to one or many skills domains through the property hasSkillsDomain to represent the skills domain(s) that it targets.
The EduProgression ontology is freely accessible online ${ }^{1}$. It is composed of 7 OWL classes and 8 OWL object properties and it is currently populated with the progressions of the French educational system for the subjects of History, Geography and Experimental Sciences and Technology and for the French school courses CE2, CM1 and CM2.

Below is an excerpt from an EKS represented and described through EduProgression.

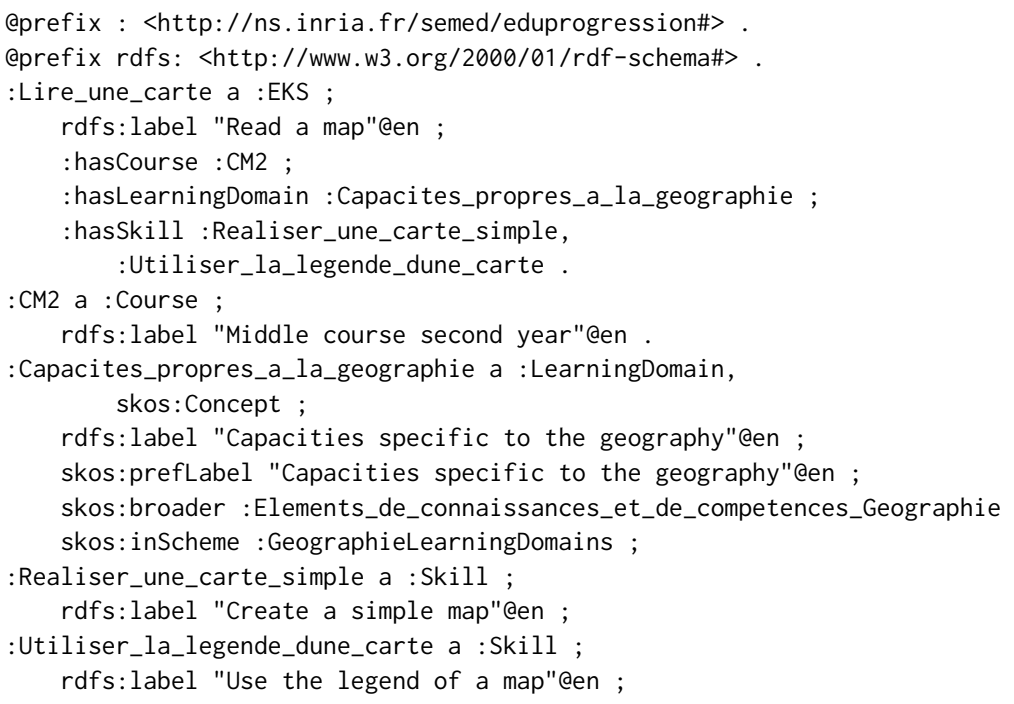

\subsection{Les Incollables Knowledge Base}

In the framework of the Intelliquiz collaborative project between Inria http://www.inria.fr and Qwant ${ }^{2}$, we aim to develop an automatic engine to generate quizzes containing multiple-choice questions, with their answers and distractors, by initially taking as a reference the topics and the information currently present in the limited dataset of questions of Les Incollables ${ }^{3}$, using the Web of Data as knowledge source.

Les Incollables is a world-famous questions game in French, targeted mainly to contribute to educate children and young people by playing. A large amount of the questions of the game are multiplechoice questions, which have been originally created manually by domain experts and written on paper cards and several nondigital formats. These questions have been created considering the different school levels of the French educational system.

As part of this project, around 160,000 questions of the game belonging to 6 French school courses (CP, CE1, CE2, CM1, CM2 and $\left.6^{e}\right)$ have been subjected to a digitization process. This digitization process included OCR from game cards, scanned documents and existing images containing the questions. Once digitized, these questions were represented and described by means of OWL ontologies and stored in a structured data format (RDF). The OWL ontologies used were:

- Linquest $^{4}$. An OWL vocabulary that describes and represents the most common elements of multiple-choice quizzes, such

\footnotetext{
${ }^{1} \mathrm{http}: / /$ ns.inria.fr/semed/eduprogression

${ }^{2}$ http://www.qwant.com

${ }^{3}$ http://www.lesincollables.com

${ }^{4}$ http://ns.inria.fr/semed/linquest
} 


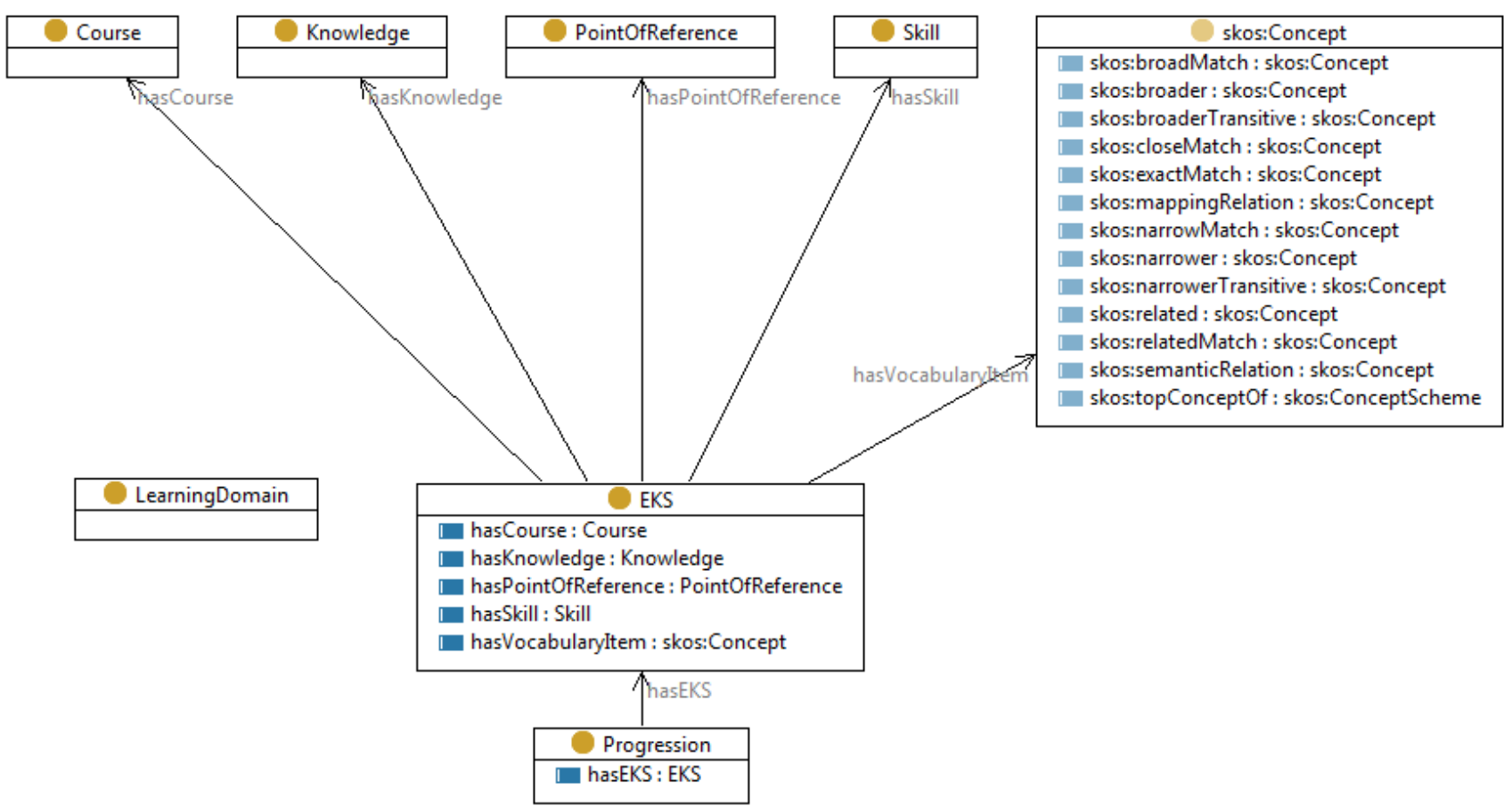

Figure 1: Classes and Properties of the EduProgression Ontology

as pair of a question and its answer QA, the natural language representation of such question NLQuestion and such answer NLAnswer, a set of questions QASet and set of sets of questions QAMultiset.

- Incollables ${ }^{5}$. An OWL vocabulary that describes and represents the most specific aspects of the quizzes of the Les Incollables game, such a the theme (subject or subjects) of a linquest set of questions (QASet) hasTheme and its level hasLevel. Also the DBpedia resource(s) related to a linquest question (QA) hasRelatedDBpediaResource.

- FrenchEd $u^{6}$. It formalizes the cycles, courses and related information of the French educational system, making possible to represent them in a standard formal model, searchable and understandable by machines. Its main classes are Degree and School.

After this, each question element QA, has been annotated automatically with its corresponding DBpedia resources by using DBpedia Spotlight ${ }^{7}$ and with some keywords. For the experiments described in this paper, we obtained a subset of the 126 most representative questions from the subject of geography, from the 6 school courses.

An excerpt of the Les Incollables Knowledge Base is shown below.

\footnotetext{
${ }^{5}$ http://www.gaya-technology.com/incollables

${ }^{6} \mathrm{http}: / /$ ns.inria.fr/semed/frenchedu/

${ }^{7}$ http://www.dbpedia-spotlight.org/
}

eprefix dbpedia-fr: <http://fr.dbpedia.org/resource/> . eprefix inc: <http://www.gaya-technology.com/incollables\#> . aprefix lq: <http://ns.inria.fr/semed/linquest\#> . aprefix fe: <http://ns.inria.fr/semed/frenchedu\#> . inc: QASet-HG-CE1 a lq:QASet ;

$l q:$ hasQA <\#Q2163>;

inc:hasLevel fe:Cours_elementaire_premiere_annee ; inc:hasTheme inc:HistoireGeoEdCiv .

inc:Q2163 a lq:QA ;

lq:NLQuestion "Quelle est la capitale de l'Espagne ?"@fr ; lq:NLQuestion "What is the capital of Spain ?"@en ; inc:hasRelatedDBpediaResource dbpedia-fr:Madrid, dbpedia-fr:Espagne, dbpedia-fr:Capitale .

\section{EXTRACTION OF A KNOWLEDGE GRAPH FROM DBPEDIA ACCORDING TO THE EDUCATIONAL STANDARDS}

The selection of resources from a dataset that are relevant to the topics of a subject for a specific school year according to an educational standard, is the basis for automatically generating useful quizzes for the learners. Since the knowledge bases on the Semantic Web use different ontologies and ways of structuring their data, we decided to focus our study on DBpedia, which is one of the most used knowledge bases available on the Linked Open Data and provides a large amount of resources from different domains.

To extract a subset from DBpedia that contains knowledge from a specific domain and a school year (to which we refer to as a 
knowledge graph), our approach is as follows: We start by analyzing the keywords of the competences and skills defined in each knowledge base of an educational standard corresponding to the targeted domain and school year. Then, from these keywords, we extract an initial set of DBpedia named entities $R$ and we enrich it with the wikilinks of the extracted resources having relevant categories. For this, we build a set of categories $C$ with the result of a dedicated SPARQL query on DBpedia searching for the value of the dcterms:subject property or dcterms:subject/skos:broader property path of each resource in $R$. Then we restrict to the subset of $C$ limited to the $k$ categories having the most related resources. We consider $C_{t o p K}$ as the set of the most relevant categories and therefore the wikilinks of the resources in $R$ which have a category in $C_{t o p K}$ are added to $R$. The value of the number of categories $k$ is determined by a manual analysis of the relevance of the categories with respect to the domain, allowing to discard some categories that may not be relevant to the targeted domain. Finally the set of triples describing the resources in $R$ are stored in a named graph NG.

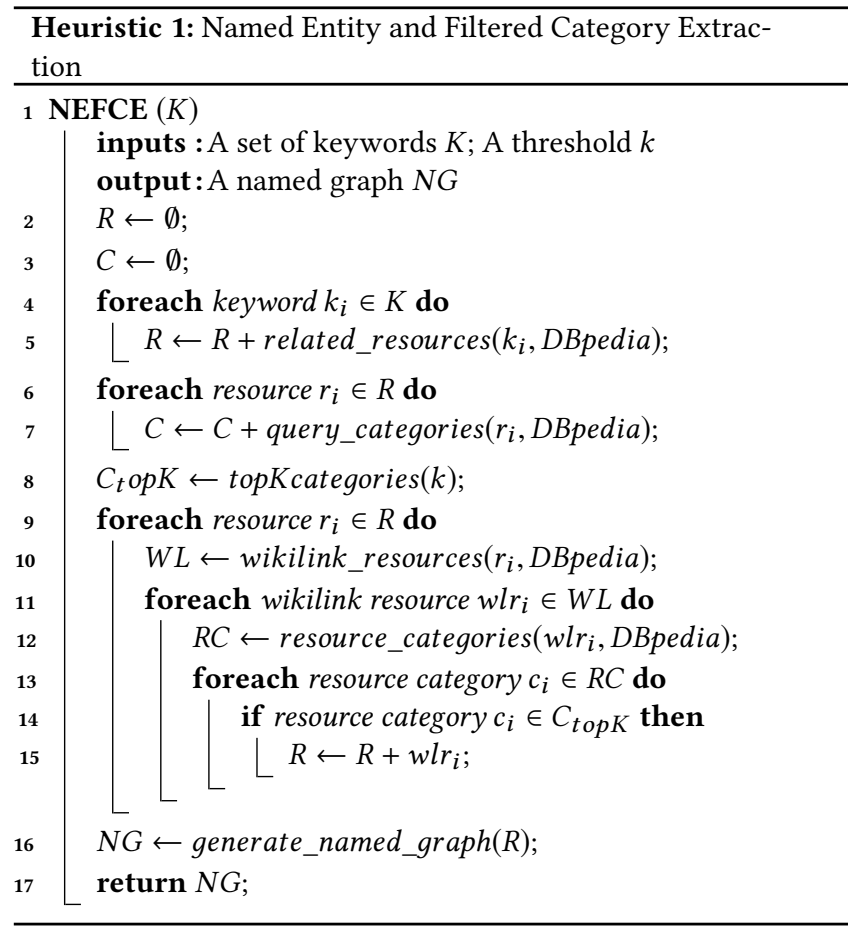

The main advantage of this heuristics is that it enables to discover relevant related resources from DBpedia and it limits the number of non-relevant resources that may be added through a category-based filter.

By means of a simple SPARQL like the one shown, it is possible to retrieve all the DBpedia resources contained in the generated knowledge graph:

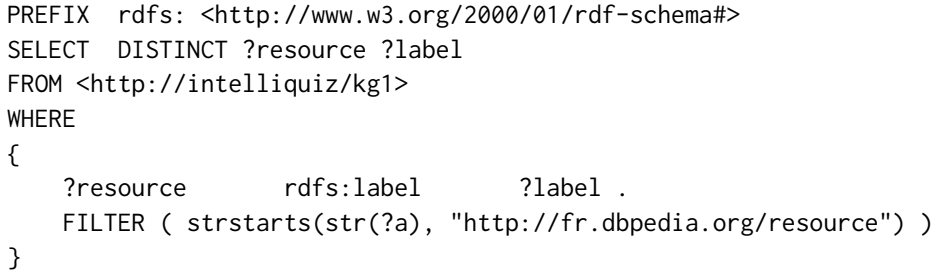

\section{AUTOMATIC GENERATION OF QUIZZES FROM A KNOWLEDGE GRAPH}

As already discussed in [15], it is considered that the generation of quizzes is a time-consuming task that can be automated by taking advantage of existing knowledge bases available on the Web. For this, we proposed an approach to automatically generate quizzes from knowledge bases on the Web of Data, that relies on the work of [14] on the generation of multiple choice questions from domain ontologies through queries. We proposed a new classification of the strategies to generate quizzes. The first column of Table 1 lists all the categories of strategies to generate quizzes. As it can be seen on this table, each category corresponds to a type of targeted question to be generated; and to achieve it, the expected output is a special kind of RDF triple which should be retrieved from a knowledge graph, by using a predefined SPARQL query template.

When considering this approach of automatic generation of quizzes, its effectiveness critically depends on the relevancy of the knowledge graph considered to answer the SPARQL queries implementing the strategies.

\section{EMPIRICAL VALIDATION OF THE PROPOSED APPROACH}

In order to comparatively evaluate the two reference standards of knowledge and skills (Les Incollables and EduProgression), we have generated a knowledge graph from the keywords defined by each one for the CM2 school year.

Then we evaluated the relevance of the resources contained in each knowledge graph. Finally we measured the impact that the resources in each knowledge graph can have on the questions generated for the quizzes.

\subsection{Evaluation of the generated knowledge graphs through the scoring of the selected resources}

In order to assess the relevance of the resources contained in the two knowledge bases for the CM2 school year of geography, we asked a geography teacher who teaches the CM2 level, to evaluate the relevance of each resource of a selected list to the domain and to the school level. The teacher was given a spreadsheet with a list of 188 resources randomly extracted from the two knowledge bases, that is, 100 resources per each base merged to avoid duplicates. Each resource was evaluated on a scale of 1 (not at all relevant) to 5 (very relevant).

Once the relevance of the resources was evaluated, we have calculated the precision of the resources generated from each reference standard. We defined it as the proportion of relevant resources 


\begin{tabular}{|c|c|c|}
\hline Category & Output & Example Question \\
\hline Find resources of a given class & 1 resource and 1 class & What is the Seine? a river \\
\hline Find a data property that relates a resource and a data value & 1 resource, 1 data value and 1 data property & What is the total population of Paris? 2240621 \\
\hline Find an object property that relates two resources & 1 property and 2 resources & In which city is the Université Nice Sophia Antipolis? in Nice \\
\hline Find superclasses of a given class & 2 classes ( 1 class and its superclass) & What is a Synagogue? a religious building \\
\hline
\end{tabular}

Table 1: Categories of strategies to generate questions from a knowledge graph and their expected outputs

\begin{tabular}{ccc}
\hline & EduProgression & Les Incollables \\
\hline Precision & 0,78 & 0,73
\end{tabular}

Table 2: Precision of the resources per educational reference

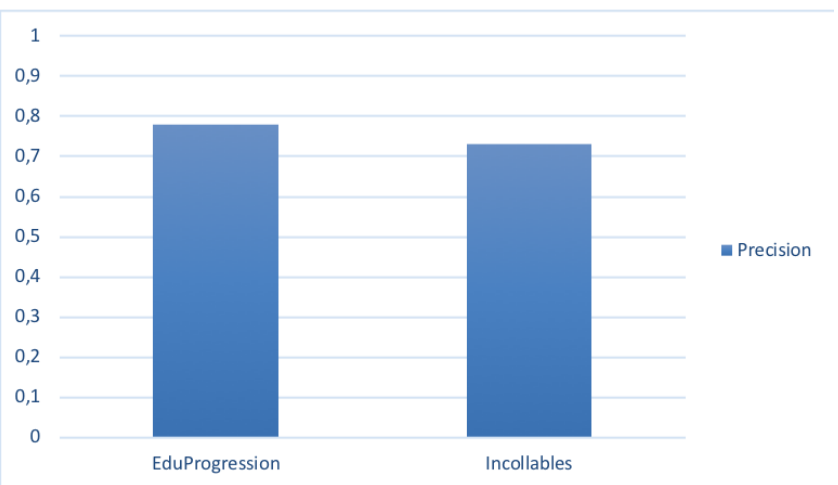

Figure 2: Precision of the resources of each reference standard

among a set of resources generated from a given reference standard (equation (1)).

$$
P=\frac{\text { Number of Relevant Resources }}{\text { Total Set of Resources Generated from a Reference Standard }}
$$

We have considered that a resource is relevant if its score is greater than or equal to 3 .

The precision obtained by the resources of each educational reference is reported in table 2 and shown in figure 2.

As we can see, after this evaluation, the resources generated from the standard of reference EduProgression were more relevant according to the domain and according to the school level.

\subsection{Evaluation of the generated knowledge graphs through the scoring of the generated quizzes}

Once the pertinence of the resources generated from the two reference educational standards has been evaluated, we have applied the quiz generation techniques mentioned in section 5 , to each of the DBpedia subgraphs generated from each reference educational standard. The number of questions generated from each subgraph is reported in table 3.

Finally, we have asked the geography teacher to evaluate the relevance of the questions generated from each educational reference

\begin{tabular}{ccc}
\hline & EduProgression & Les Incollables \\
\hline Questions & 191 & 286
\end{tabular}

Table 3: Number of generated questions per educational reference

\begin{tabular}{ccc}
\hline & EduProgression & Les Incollables \\
\hline Average relevance & 3.25 & 2.87
\end{tabular}

Table 4: Average relevance of the questions per educational reference

(on a scale of one to 5 , where 5 is the most relevant). For this, he has been given a list of 100 questions for each educational reference, extracted randomly.

The results of this evaluation are shown below in table 4 .

From these results it can be interpreted that the questions generated from the DBpedia resources extracted from the educational reference "EduProgression" were evaluated with a higher average score of relevance with respect to "Les Incollables". These results are consistent with the assessed relevance of the evaluated resources extracted from each educational reference.

\section{CONCLUSIONS AND FUTURE WORK}

In this paper, we have presented an approach to the automatic generation of quizzes about a specific subject, that takes into a account the school level of the target student. Through an educational reference, our approach is able to extract resources from the LOD (corresponding to a specific subject and a specific school year) from which the quizzes are generated. We have presented two structured educational references: EduProgression and Les Incollables and we have evaluated them in order to determine from which one it is possible to extract more relevant resources and quizzes. From the evaluation made by a geography teacher to resources and quizzes of geography for the CM2 school level, we were able to conclude that EduProgression was the educational reference that allowed generating resources and quizzes of greater relevance. However, as future work, we plan to extend our evaluation by taking into account quizzes generated for other school courses and subjects, and also by involving more professors. Future work also includes extracting resources from other linked data sources and not just from DBpedia. Finally, we think about improving our heuristic for the selection of related resources, also giving the possibility to the generator of quizzes, to manually select the categories from which to filter the related resources. 


\section{REFERENCES}

[1] [n. d.]. Le Socle Commun de Connaissances et de Compétences. ([n. d.]). http: //media.education.gouv.fr/file/46/7/5467.pdf

[2] Asma Ben Abacha, Júlio Cesar dos Reis, Yassine Mrabet, Cédric Pruski, and Marcos Da Silveira. 2016. Towards natural language question generation for the validation of ontologies and mappings. F. Biomedical Semantics 7 (2016), 48 https://doi.org/10.1186/s13326-016-0089-6

[3] Naveed Afzal and Ruslan Mitkov. 2014. Automatic generation of multiple choice questions using dependency-based semantic relations. Soft Computing 18, 7 (2014), 1269-1281. https://doi.org/10.1007/s00500-013-1141-4

[4] M. Al-Yahya. 2011. OntoQue: A Question Generation Engine for Educational Assesment Based on Domain Ontologies. In 2011 IEEE 11th International Conference on Advanced Learning Technologies. 393-395. https://doi.org/10.1109/ ICALT.2011.124

[5] Maha Al-Yahya, Auhood Al-Faries, and Remya George. 2013. CURONTO: An Ontological Model for Curriculum Representation. In Proceedings of the 18th ACM Conference on Innovation and Technology in Computer Science Education (ITiCSE '13). ACM, New York, NY, USA, 358-358. https://doi.org/10.1145/2462476.2465602

[6] Tahani Alsubait, Bijan Parsia, and Uli Sattler. 2014. Generating Multiple Choice Questions From Ontologies: Lessons Learnt. In Proceedings of the 11th International Workshop on OWL: Experiences and Directions (OWLED 2014) co-located with 13th International Semantic Web Conference on (ISWC 2014), Riva del Garda, Italy, October 17-18, 2014. 73-84. http://ceur-ws.org/Vol-1265/owled2014_submission_ 11.pdf

[7] Tahani Alsubait, Bijan Parsia, and Uli Sattler. 2015. Generating Multiple Choice Questions From Ontologies: How Far Can We Go? Springer International Publishing, Cham, 66-79. https://doi.org/10.1007/978-3-319-17966-7_7

[8] Tahani Alsubait, Bijan Parsia, and Ulrike Sattler. 2016. Ontology-Based Multiple Choice Question Generation. KI - Künstliche Intelligenz 30, 2 (2016), 183-188. https://doi.org/10.1007/s13218-015-0405-9

[9] M. Bucos, B. Dragulescu, and M. Veltan. 2010. Designing a semantic web ontology for E-learning in higher education. In Electronics and Telecommunications (ISETC),
2010 9th International Symposium on. 415-418. https://doi.org/10.1109/ISETC. 2010.5679298

[10] Marija Cubric and Milorad Tosic. 2010. Towards automatic generation of eassessment using semantic web technologies. In Proceedings of the 2010 International Computer Assisted Assessment Conference. http://hdl.handle.net/2299/4885

[11] Hilary Dexter and Ioan Davies. 2009. An Ontology-Based Curriculum Knowledgebase for Managing Complexity and Change. 2014 IEEE 14th International Conference on Advanced Learning Technologies 0 (2009), 136-140. https: //doi.org/10.1109/ICALT.2009.85

[12] Muriel Foulonneau. 2012. Generating Educational Assessment Items from Linked Open Data: The Case of DBpedia. Springer Berlin Heidelberg, Berlin, Heidelberg, 16-27. https://doi.org/10.1007/978-3-642-25953-1_2

[13] Cui Guangzuo, Chen Fei, Chen Hu, and Li Shufang. 2004. OntoEdu: a case study of ontology-based education grid system for e-learning. In GCCCE International conference, Hong Kong.

[14] Andreas Papasalouros, Konstantinos Kanaris, and Konstantinos Kotis. 2008. Automatic Generation Of Multiple Choice Questions From Domain Ontologies.. In e-Learning (2009-03-01), Miguel Baptista Nunes and Maggie McPherson (Eds.). IADIS, 427-434. http://dblp.uni-trier.de/db/conf/iadis/el2008.html\# PapasalourosKK08

[15] O. Rodriguez Rocha and C. Faron Zucker. 2017. AUTOMATIC GENERATION OF EDUCATIONAL QUIZZES FROM DOMAIN ONTOLOGIES. In EDULEARN17 Proceedings (9th International Conference on Education and New Learning Technologies). IATED, 4024-4030. https://doi.org/10.21125/edulearn.2017.1866

[16] Oscar Rodríguez Rocha, Catherine Faron Zucker, and Geraud Fokou Pelap. 2017. A Formalization of the French Elementary School Curricula. In Knowledge Engineering and Knowledge Management, Paolo Ciancarini, Francesco Poggi, Matthew Horridge, Jun Zhao, Tudor Groza, Mari Carmen Suarez-Figueroa, Mathieu d'Aquin, and Valentina Presutti (Eds.). Springer International Publishing, Cham, 82-94.

[17] Maha Al Yahya, Remya George, and Auhood Alfaries. 2015. Ontologies in ELearning: Review of the Literature. International fournal of Software Engineering and Its Applications 9, 2 (2015), 67-84. 